\title{
Journal of Sports Medicine \& Doping Studies
}

\section{Understanding the Fatigue-Recovery Cycle in Team Sport Athletes}

\author{
Luke W. Hogarth ${ }^{\star}$, Brendan J. Burkett and Mark R. McKean \\ School of Health and Sport Sciences, University of the Sunshine Coast, Australia
}

*Corresponding author: Luke W. Hogarth, School of Health and Sport Sciences, University of the Sunshine Coast, Locked Bag 4, Maroochydore DC, Queensland, 4558, Australia, Tel: +61 75456 5402; Fax: +61 75459 4600; Email: luke.hogarth@research.usc.edu.au

Rec date: Nov 24, 2014, Acc date: Nov 26, 2014, Pub date: Nov 28, 2014

Copyright: @ 2015 Hogarth LW, et al. This is an open-access article distributed under the terms of the Creative Commons Attribution License, which permits unrestricted use, distribution, and reproduction in any medium, provided the original author and source are credited.

\section{Editorial}

Identifying and understanding the fatigue response of athletes following intensive training and competition is necessary to avoid injury, performance decrements and overtraining. There is an abundance of tests and monitoring tools that have been used to describe the fatigue response of both individual- and team-sport athletes. These tests typically include measures of neuromuscular function, subjective questionnaires, blood and salivary markers, or physical performance tests. Importantly though, the tests employed must be valid, reliable and practically convenient in applied settings. Investigators have commonly assessed fatigue measures during tournament competition [1-3] or over subsequent days following competitive matches [4-6] in an attempt to identify the time course of the fatigue-recovery cycle. This provides an understanding of how an athlete recovers from competition and guides training loads and recovery practices when athletes may be most vulnerable to nonfunctional overreaching and injury.

Measures of neuromuscular function have commonly been used to examine the fatigue response following team sport activities and have been suggested to be reliable indicators of low-frequency fatigue $[7,8]$. Neuromuscular function has been assessed using jumping protocols, sprinting performance, and isokinetic and isointertial dynamometry. Perhaps the most commonly utilised are jumping tests, as they incorporate the stretch-shortening capability of the lower body musculature and are easy to administer. Measures of both upper- and lower-body neuromuscular function such as mean power and force production, time to peak force and jump height and have shown acceptable reliability and day-to-day variability making them useful measures to assess fatigue over a training mesocycle or playing season [9-11]. Team sport athletes competing in contact sports such as rugby football can show severe reductions in both upper- and lower-body neuromuscular function following match-play suggested to be caused by reductions in contraction velocity associated with increased muscle damage [12-14]. Other studies examining neuromuscular fatigue in low or non-contact team sports have questioned the sensitivity of jumping-derived measures $[1,15]$. However, the degree of jump performance decrement has been associated with match activities such as maximal decelerations that likely cause exercise-induced muscle damage and increases in general muscle soreness in these sports [1]. Further, decreased jump performance has been associated with reduced physical and technical performance during subsequent matchplay suggesting that jump-derived measures provide a valid assessment of neuromuscular fatigue for team sport athletes $[1,16,17]$.

Subjective questionnaires are another simple and cost-effective tool to assess the fatigue-recovery cycle following competition and training activities. There are a number of questionnaires that have been used in the literature such as the POMS, RESTQ-Sport and DALDA questionnaires $[8,18]$. Studies have shown alterations in perceptual fatigue to be more sensitive to tournament competition than jumpderived measures of neuromuscular fatigue [1] and perceived fatigue and muscle soreness are known to outlast suppressed neuromuscular function and biochemical markers following rugby league matches [14]. Increases in perceptual fatigue are suggested to alter an athlete's sense of effort and force them to down-regulate their exercise capacity [19]. Indeed, players with low perceptual well-being prior to tournament competition regulate their high-intensity activity in the earlier matches perhaps to better maintain performance during the latter stages of the tournament where finals matches are typically played [1]. Therefore, assessing perceptual fatigue prior to and following competition may be useful to avoid performance decrements during subsequent matches and training activities. There is concern over the subjectivity of questionnaires as athletes may manipulate their response to gain a favourable outcome and scores may be influenced by factors such as the match outcome, poor player compliance due to tedious daily measures, and diurnal fluctuations $[8,18]$. Nevertheless, the cost-effective and time efficient use of subjective questionnaires provides coaches and sport scientists with a useful assessment of an athlete's fatigue-recovery cycle, particularly when they do not have access to specialist training facilities or equipment.

Blood and salivary markers have been used to examine the possible mechanisms associated with neuromuscular and perceptual fatigue following team sport competition $[7,14,16]$. Although testing samples can be expensive, changes in biochemical, hormonal and immunological measures can be easily assessed around daily training activities with non-invasive blood capillary samples and salivary swabs. Measures such as creatine kinase, salivary cortisol and testosterone concentrations, plasma glutamine and salivary immunoglobulin A are commonly cited in the literature as markers used to examine athletes health status and response to intensive training and competition $[8,18]$. However, researchers have found poor temporal relationships with biochemical markers and neuromuscular function following competition and it is yet to be determined if a single biochemical, hormonal or immunological measure can be used to detect non-functional overreaching or overtraining in athletes $[7,18]$. Further, some biochemical measures such as creatine kinase have been shown to have high day-to-day variability questioning whether these measures can be used to examine changes in an athlete's health status or fatigue-recovery cycle [8]. Given the costly expense of obtaining blood and salivary measures in team sport settings practitioners should take careful consideration when deciding to use these measures for longitudinal athlete assessment. However, they may provide a useful diagnostic tool for identifying the mechanisms of fatigue when athletes may be at risk of non-functional overreaching, sickness or injury.

By developing a strategic plan aimed at understanding the fatiguerecovery cycle following intensive training and competition an athlete's health status and physical performance can be effectively managed. 
Whilst several neuromuscular, perceptual and blood and salivary measures can be used to describe the fatigue-recovery cycle following team sport activity, a combination of two or more of these measures may provide the most conclusive insights for longitudinal athlete assessments. It is critical that the selected measures are cost-effective, unobtrusive and easy to administer around daily training activities. Further, practitioners need to be aware of the day-to-day variability of measures in order to make meaningful interpretations from the data.

\section{References}

1. Hogarth LW, Burkett BJ, McKean MR (2014) Neuromuscular and Perceptual Fatigue Responses to Consecutive Tag Football Matches. Int J Sports Physiol Perform .

2. McNamara DJ, Gabbett TJ, Naughton G, Farhart P, Chapman P (2013) Training and competition workloads and fatigue responses of elite junior cricket players. Int J Sports Physiol Perform 8: 517-526.

3. West DJ, Cook CJ, Stokes KA, Atkinson P, Drawer S et al. (2014) Profiling the time-course changes in neuromuscular function and muscle damage over two consecutive tournament stages in elite rugby sevens players. J Sci Med Sport 17: 688-692.

4. Cormack SJ, Newton RU, McGuigan MR (2008) Neuromuscular and endocrine responses of elite players to an Australian rules football match. Int J Sports Physiol Perform 3: 359-374.

5. McLean BD, Coutts AJ, Kelly V, McGuigan MR, Cormack SJ (2010) Neuromuscular, endocrine, and perceptual fatigue responses during different length between-match microcycles in professional rugby league players. Int J Sports Physiol Perform 5: 367-383.

6. McLellan CP, Lovell DI, Gass GC (2010) Creatine kinase and endocrine responses of elite players pre, during, and post rugby league match play. J Strength Cond Res 24: 2908-2919.

7. McLellan CP, Lovell DI, Gass GC (2011) Markers of postmatch fatigue in professional Rugby League players. J Strength Cond Res 25: 1030-1039.
8. Twist C, Highton J (2013) Monitoring fatigue and recovery in rugby league players. Int J Sports Physiol Perform 8: 467-474.

9. Cormack SJ, Newton RU, McGuigan MR, Doyle TL (2008) Reliability of measures obtained during single and repeated countermovement jumps. Int J Sports Physiol Perform 3: 131-144.

10. Hogarth LW, Deakin G, Sinclair W (2013) Are Plyometric Push-Ups a Reliable Power Assessment Tool? Journal of Australian Strength \& Conditioning 21(Supplement 1): 67-69.

11. McLellan CP, Lovell DI, Gass GC (2011) The role of rate of force development on vertical jump performance. J Strength Cond Res 25: 379-385.

12. Johnston RD, Gabbett TJ, Jenkins DG (2013) Influence of an intensified competition on fatigue and match performance in junior rugby league players. J Sci Med Sport 16: 460-465.

13. McLellan CP, Lovell DI (2012) Neuromuscular responses to impact and collision during elite rugby league match play. J Strength Cond Res 26: 1431-1440.

14. Twist C, Waldron M, Highton J, Burt D, Daniels M (2012) Neuromuscular, biochemical and perceptual post-match fatigue in professional rugby league forwards and backs. J Sports Sci 30: 359-367.

15. Krustrup P, Zebis M, Jensen JM, Mohr M (2010) Game-induced fatigue patterns in elite female soccer. J Strength Cond Res 24: 437-441.

16. Johnston RD, Gibson NV, Twist C, Gabbett TJ, MacNay SA, et al. (2013) Physiological responses to an intensified period of rugby league competition. J Strength Cond Res 27: 643-654.

17. Mooney MG, Cormack S, OÊ1/4brien BJ, Morgan WM, McGuigan M (2013) Impact of neuromuscular fatigue on match exercise intensity and performance in elite Australian football. J Strength Cond Res 27: 166-173.

18. Robson-Ansley PJ, Gleeson M, Ansley L (2009) Fatigue management in the preparation of Olympic athletes. J Sports Sci 27: 1409-1420.

19. Marcora SM, Staiano W, Manning V (2009) Mental fatigue impairs physical performance in humans. J Appl Physiol (1985) 106: 857-864. 